\title{
VIGÉSIMO ANIVERSARIO DE LA LEY DE CONCILIACIÓN DE LA VIDA LABORAL Y FAMILIAR
}

\section{TWENTIETH ANNIVERSARY OF THE LAW OF CONCILIATION OF LABOR AND FAMILY LIFE}

José María Uris Lloret

joseull@outlook.es

Resumen Se cumplen veinte años desde que se aprobó la Ley 39/1999, de 5 de noviembre, para promover la conciliación de la vida laboral y familiar de las personas trabajadoras. El presente artículo tiene como objetivo analizar los avances que se han producido y el camino que aún nos queda por recorrer en la materia que nos ocupa, pero en lugar de tomar como referencia el año noventa y nueve, consideramos que es más apropiado ubicarnos hace aproximadamente cinco décadas, que es cuando las mujeres se fueron incorporando de forma masiva al trabajo remunerado.

Entendemos que en dicho periodo los progresos no han sido significativos, como se desprende de que aún pervive un modelo desfasado, donde la mayor parte de las tareas del hogar aún las asumen las mujeres y que los empleadores no facilitan el equilibrio de la vida laboral, familiar y personal, sino más bien todo lo contrario.

Desde ese escenario tan negativo partimos para hacer frente al déficit tan importante que hay en conciliación y a los nuevos desafíos que nos depara el futuro, donde habrá que invertir muchos recursos en las tareas de cuidado, cuando nos dirigimos hacia una nueva era económica que suscita muchas incógnitas.

Palabras clave Conciliación, igualdad, modelo desfasado, género, discriminación.

\begin{abstract}
Twenty years have passed since Law 39/ 1999 of November 5th was approved to promote the conciliation of working and family life of working people. The objective of this article is to analyze the advances that have taken place and the path that we have to walk in this matter, but instead of taking as reference the ninetyninth year, we consider that is more appropiate to locate ourselves five decades ago approximately, when women were incorporated to remunerated work.

We understand that in that period progress hasn't been significant, as it is understood that there is still an out of phase model, where most of housework are still assumed by women and employers don't facilitate the balance of working life, family and personal, rather the complete opposite.

From this negative stage we split to face the important deficit that exists in conciliation and the new challenges than the future offer to us, where many resources will have to be invested in tasks of care, when we go to a new economic era that offers many unknowns.
\end{abstract}

Keywords Conciliation, equality, out of phase model,gender, discrimination. 


\section{Introducción: un lento cambio de modelo}

Se cumplen ahora veinte años desde que se aprobó la Ley 39/1999, de 5 de noviembre, para promover la conciliación de la vida laboral y familiar de las personas trabajadoras. Seguramente que, con motivo del tal evento, se realizarán innumerables jornadas y se publicarán diversos estudios y artículos analizando los avances que se han producido en dicho período y el camino que aún nos queda por recorrer.

Con esa finalidad, el presente artículo también tiene como objetivo analizar lo que se ha progresado en el tema que nos ocupa, pero en lugar de tomar como referencia el año noventa y nueve cuando se aprobó la Ley de Conciliación, se considera más apropiado ubicarnos hace aproximadamente cinco décadas, que es cuando las mujeres se fueron incorporando de forma masiva al trabajo remunerado, sin que ni sus parejas ni las instituciones las apoyaran en las labores de cuidado que venían realizando habitualmente, convirtiéndose la conciliación en un problema de ellas, cuando en la realidad era y es una cuestión que debe asumir el conjunto de la sociedad.

Ese déficit, en un asunto tan transcendental para toda la ciudadanía, se ha puesto de manifiesto en las dificultades que tienen las parejas de doble ingreso, prácticamente hoy todas (debido a la carestía de la vida y a los bajos salarios, son necesarias todas las aportaciones económicas), las familias monomarentales y monoparentales, para lograr un equilibrio entre la vida laboral, familiar y personal.

Pero no solo el tema económico incide para que mujeres y hombres, tengan entre sus prioridades acceder al mercado de trabajo, en este último medio siglo, que se ha caracterizado por los profundos cambios que han tenido lugar en todos los aspectos, uno de los más importantes, sin duda, es que un importante número de personas aspiran a construirse un proyecto de vida, sin que el mismo venga impuesto por los usos sociales, atendiendo a su sexo $u$ otras circunstancias.

Porque las personas construimos nuestras vidas a través de diversos proyectos, y siguiendo nuestra vocación pretendemos darle sentido a la misma. Las aspiraciones personales nos muestran que los hombres y las mujeres no sólo se mueven en el ámbito laboral y familiar, sino que también necesitan tiempo para sí mismos, para sus intereses y sus aficiones, por eso debemos considerar los tres dominios en que normalmente se realiza la persona: el trabajo, la familia y el mundo más personal de la misma. Y para llevar a cabo nuestras pretensiones precisamos tener espacios de libertad importantes, y sin lugar a duda, necesitamos una remuneración económica para conseguir los objetivos que nos hemos propuesto.

Ya que la independencia económica que nos proporciona un empleo, en cierta medida, nos permite abordar otras áreas que tenemos interés en conocer y poder disponer de un tiempo para el descanso y para el ocio. Así, para poder llevar a cabo con eficacia las tareas que debemos realizar, tanto en el ámbito privado como en el público, deberemos estar en buenas condiciones físicas y psíquicas, y según la Organización Mundial de la Salud, la salud es un estado de completo bienestar físico, mental y social, y no solamente la ausencia de enfermedades o afecciones. 
A este respecto Valls-Llobet (2010) introduce el concepto de salud para disfrutar 0 salud para vivir, donde se entiende:

Un nuevo concepto de salud ligado a la potenciación de la energía de las personas, a mejorar su rendimiento físico e intelectual subsanando la mayoría de las carencias metabólicas o las disfunciones endocrinológicas. Un concepto de salud relacionado con la calidad de vida, con la capacidad de trabajar en mejores condiciones. (p. 26)

Difícilmente podremos realizar labores de cuidado y realizar nuestro trabajo, si no nos ocupamos de nuestro bienestar.

Y una parte fundamental de nuestro bienestar, es que nuestro cometido sea de utilidad para el resto de la sociedad, tanto cuando lo realizamos desde un empleo retribuido económicamente, como cuando nuestras iniciativas en otras áreas aportan beneficio a terceras personas, por ejemplo las tareas de voluntariado, que en la actualidad ocupan a un número importante de ciudadanas y ciudadanos; habitualmente cuando ves reportajes sobre la labor de los voluntarios o cuando hablas con alguno de esas personas, generalmente cuentan que reciben mucho más de lo que aportan, la satisfacción que sienten por haber cooperado en proyectos de solidaridad, les compensa en mucho el esfuerzo realizado.

No se está investigando, con la amplitud que requiere el tema, los beneficios que está aportando a la sociedad y en consecuencia el progreso que aporta a la ciudadanía el ir dejando atrás, poco a poco y con excesiva lentitud, un modelo patriarcal que tenía sentados sus cimientos en una división sexual del trabajo donde los varones se dedicaban a las tareas de producción y las mujeres a las de reproducción, a un modelo igualitario e inteligente donde todas las personas participan en la misma medida, independientemente de su sexo.

Con el tiempo, probablemente vayamos conociendo la magnitud de los avances y los beneficios que nos ha proporcionado el que mujeres y hombres trabajen en igualdad. Ganancia para el conjunto de la sociedad por lo que nos aporta el nuevo modelo, y mejora para sus integrantes, porque se sentirán más realizados al ser de utilidad para la ciudadanía y poder cuidar y convivir más tiempo con su familia. No obstante, como se ha indicado anteriormente, el camino no es fácil, más bien largo y tortuoso, y materias, como la que estamos analizando en este artículo, que son imprescindibles superar para lograr el objetivo de una sociedad igualitaria, se presentan muy complejas y de difícil solución, como veremos en los siguientes epígrafes.

\section{La evolución de la sociedad como condicionante de la conciliación}

La sociedad, como es natural, va evolucionando, no sería lógico que se mantuviera estática, y a su vez los usos y costumbres presentes en la misma, van cambiando. Esa trayectoria que durante siglos ha mantenido una evolución bastante uniforme, aunque en algunos periodos históricos el proceso ha sido nulo o incluso ha retrocedido, en el último medio siglo ha tenido un progreso muy significativo, que ha 
modificado profundamente la percepción y los conceptos sobre determinados aspectos sociales y también las formas de vida de las personas.

En gran medida, esas transformaciones tienen su causa en los grandes avances tecnológicos que han tenido lugar en la sociedad de la comunicación e información. Las nuevas tecnologías han originado transcendentales cambios en las organizaciones productivas y en la forma de desenvolverse la ciudadanía. Actualmente el número de líneas móviles, que además son ordenadores de bolsillo y se puede acceder a internet, entre otras muchas prestaciones, supera a la población mundial.

Por lo que es manifiesto, lo condicionado que está el desenvolvimiento de las personas por las nuevas tecnologías, pero también hay otros fenómenos como la globalización y los medios de comunicación que influyen en nuestra manera de pensar, obrar y observar nuestro entorno.

Por lo tanto, no estamos en el mismo escenario que en la década de los sesenta del siglo anterior, cuando las mujeres empezaron a entrar en el mercado de trabajo de forma más numerosa, en el último medio siglo hemos pasado de un modelo productivo, donde la mayor parte de la población trabajaba en el sector primario, a una estructura de empleo donde la mayoría de las personas está empleada en el sector servicios.

Ese cambio tan radical en el ámbito productivo, que ha modificado profundamente las relaciones laborales, ha ido correlativo al tránsito de una sociedad rural, de base agraria, a una comunidad urbana moderna, donde la vida cotidiana de las personas y sus hábitos, son muy distintos al modelo milenario que tenía su origen en la vida campesina.

Los hechos expuestos en los párrafos anteriores han dado lugar a un nuevo entorno social, donde mujeres y hombres tienen una mentalidad muy distinta a la de generaciones anteriores $y$, por lo tanto, sus inquietudes y aspiraciones, son también otras. Encontrar un empleo y que el mismo sea de calidad es, entre otras pretensiones, uno de los proyectos a los que las personas dedican más tiempo y esfuerzo, y no pueda quedar vacía esa inversión tan importante, por no encontrar modelos de conciliación que permitan el equilibrio entre la vida laboral, familiar y personal.

Porque, como hemos manifestado anteriormente, los cambios han sido muy significativos en los últimos años, por lo tanto, las tareas de cuidado de las personas dependientes y la atención al hogar, debe tener otros planteamientos muy distintos a los que tuvieron en el pasado, ya que las estructuras familiares se han ido adaptando a la evolución que se originaban en la sociedad $y$, en consecuencia, su transformación también ha sido profunda.

Además de que por motivos laborales $u$ otros, en muchas situaciones, los miembros de una familia no residen en la misma ciudad y de esa manera no pueden tener el apoyo de sus parientes más cercanos. Igualmente, las trabajadoras y los trabajadores con frecuencia tienen el lugar de trabajo en otro municipio distinto al de su residencia y les resulta más difícil poder atender sus responsabilidades familiares.

Asimismo, también han surgido nuevas formas familiares, "junto a un tipo de familia que continúa el patrón característico del siglo XIX y que encaja bien con el 
Código Civil, han surgido multitud de situaciones afectivas y convivenciales novedosas que dibujan el nuevo rostro de las familias españolas" (Durán, 2015, p. 39). En consecuencia, las relaciones intrafamiliares han sufrido profundos cambios que afectan a la colaboración que podían realizar los integrantes del hogar familiar.

\section{Pervivencia de un modelo familiar desfasado}

Aunque en las últimas décadas se han publicado un número significativo de trabajos y estudios sobre la materia objeto de examen, y con frecuencia se difunden en los diarios noticias sobre los problemas que tienen las trabajadoras y los trabajadores para poder conciliar, encuestas como la que realizo Sigma Dos con motivo del Día Internacional de la Mujer en el año 2018, donde se recogía que el 36,5\% de los hombres abandona las tareas del hogar cuando deja de vivir solos, que un $10 \%$ más de mujeres $(79 \%)$ que de hombres $(69 \%)$ hace habitualmente la compra de alimentos y de otros productos de la casa; un $29 \%$ más de mujeres (79\%) que de hombres (50\%) cocina; un 35\% más de mujeres $(79 \%)$ que de hombres $(45 \%)$ hace la limpieza del hogar, y un $44 \%$ más de mujeres (70\%) que de hombres (26\%) plancha la ropa $^{1}$, nos reflejan nítidamente de quien lleva el peso del hogar.

Igualmente, parecidos datos muestran una encuesta realizada por el Centro de Investigaciones Sociológicas un año antes, donde se expone que aproximadamente solo dos de cada diez hombres comparten en igualdad con su pareja las tareas del hogar como cocinar, fregar los platos, limpiar la casa y hacer la compra².

Si estos datos tan decepcionantes, en relación con las tareas del hogar, evidencian que aún seguimos viviendo en un modelo desfasado, el estudio realizado por la profesora Mireia Las Heras (2018) desde el Centro Internacional Trabajo y Familia del IESE que ha sido presentado en 2018 y que nos indica que un $73 \%$ de empleados en España cree que su entorno laboral dificultad ocasional o sistemáticamente la conciliación entre trabajo y vida personal y familiar, nos manifiesta que en el mundo del trabajo, los avances no han sido significativos, sino más bien todo lo contrario.

Si eso ocurre en el ámbito familiar y en el productivo, que son los pilares fundamentales que tienen que sustentar y cooperar para que se avance en el equilibrio de la vida laboral, familiar y personal y que la conciliación no siga siendo un problema de ellas, para lograr el objetivo de igualdad entre mujeres y hombres, podemos afirmar que el papel de las Administraciones Públicas en esta materia sigue siendo muy deficitario, bien porque no ponen en marcha todas las medidas necesarias, bien porque no toman las iniciativas adecuadas.

Parece en este aspecto, como en otros tantos, que vivamos de espaldas a la realidad, y que desde las Administraciones Públicas y empresas no se sepan valorar los

1 Datos extraídos del artículo: Álvarez, R. J. (7 de marzo de 2018). El 36\% de los hombres deja las tareas del hogar al vivir en pareja. El Mundo. Recuperado de

https://www.elmundo.es/espana/2018/03/07/5a9f034d22601dc03e8b4592.html

2 Datos extraídos del artículo: (6 de junio de 2017). Solo dos de cada diez hombres comparten en igualdad las tareas de limpiar y cocinar. Más del 60\% de las mujeres españolas realizan siempre o casi siempre solas esas tareas domésticas; los hombres dedican sus labores a "pequeñas reparaciones de la casa". El País. Recuperado de https://elpais.com/politica/2017/06/06/actualidad/1496749144_438408.html 
beneficios que se obtendrían si se incorporara la conciliación de la vida laboral y familiar a las estrategias de las organizaciones y a las políticas públicas. Porque al mejorarse la calidad de vida de las familias, se optimizarían los rendimientos del trabajo. Es un hecho constatado que la satisfacción de las personas empleadas incrementa la productividad.

Lo peor que le puede suceder a una empresa es tener empleadas a personas que no se identifican con la misma y realizan deficientemente sus tareas. La mayor parte del empleo está ocupada en el sector servicios y el éxito de las sociedades mercantiles que realizan su actividad en ese sector, depende en gran medida de las capacidades mentales y cognitivas de sus trabajadores, tales como creatividad, relaciones y habilidades emocionales. Difícilmente unos trabajadores descontentos pueden trasladar una buena imagen de la entidad mercantil o de la organización productiva a los clientes y usuarios que atiendan. Igualmente, una ciudadanía que percibe que los poderes políticos no se implican en proyectos de progreso y bienestar, sienten rechazo hacia los mismos.

Siguiendo con lo expuesto en los párrafos anteriores, a la insuficiente ayuda que tienen las trabajadoras por parte de sus parejas en las tareas domésticas y a la falta de apoyos por parte de las empresas y de la Administración, también hay que adicionarle un mercado de trabajo muy difícil, en donde la precariedad amenaza con frustrar las carreras profesionales y en consecuencia los proyectos de vida.

Todo ese cumulo de circunstancias negativas, pueden afectar a la salud mental de las personas que las sufren y de esta forma lo refería Espido Freire (2008):

Señalando que: pese a los intentos por encontrar tiempo propio, las terapias, la certeza de que el nuevo modelo de mujer atrapa como en una ratonera, pese a los mensajes sociales, las ofertas de ocio, los electrodomésticos, las facilidades de la comida precocinada, el control de su fecundidad, el derecho a voto, a la propiedad, pese a que la vida nunca le resultó, en apariencia, más rica ni más sencilla, la mujer joven española sufre de una pobre salud mental. (p. 86)

El citado artículo está fechado en 2008 , cuando apenas se había iniciado la crisis económica más devastadora desde la Gran Depresión. Todos conocemos el gran impacto que ha tenido en el mundo del trabajo, las pérdidas tan importantes de derechos de las personas trabajadoras y la merma en sus salarios que se ha producido, por lo que además de que es muy urgente reestablecer las condiciones de trabajo a niveles precrisis, tampoco se pueden demorar los estudios de cómo han afectado esos tiempos difíciles a la salud de la población.

Porque la recesión económica tan devastadora que hemos vivido recientemente, además de perjudicar de forma muy notoria los derechos de las trabajadoras y trabajadores, como hemos expuesto en las líneas anteriores, también han demorado o paralizado los avances que se estaban realizando para lograr una sociedad igualitaria, por lo que es inaplazable observar lo que ha sucedido en esos años, e intentar recuperar el tiempo perdido. 


\section{Retos actuales}

Como hemos expuesto en el epígrafe anterior, el mercado de trabajo español es muy difícil, extraordinariamente complejo, altamente competitivo y con muchos estereotipos. Entre esos estereotipos que se han situado en el ámbito laboral y que perjudican al conjunto de las personas empleadas, pero especialmente a las trabajadoras, es que las labores de cuidado las realizan principalmente las mujeres y que la edad es un grave inconveniente para encontrar trabajo, especialmente para las personas mayores de cuarenta y cinco años.

Los estereotipos tienen la propiedad de marginar a las personas, y en una sociedad que tiene aspiraciones de avanzar en democracia y que entre los objetivos de desarrollo del milenio está lograr la igualdad de género y la autonomía de las mujeres, no puede seguir manteniendo estereotipos que perjudiquen a la clase trabajadora, más cuando algunas entidades mercantiles jubilan a sus altos directivos a edades muy avanzadas. Porque a la discriminación que sufren como mujer, se suma la marginación por ser madre y la exclusión por edad.

En relación con lo que hemos expuesto al inicio del presente epígrafe, al recaer las labores de cuidado, principalmente en las mujeres, como se observa en todas las estadísticas, incide que durante los años que sus hijos necesitan más atención se interrumpe su carrera profesional. Cuando los hijos son menos dependientes, debido a que las mujeres en nuestro país, por todas las circunstancias que les acontecen, son las que más retrasan la maternidad en el conjunto de la Unión Europea teniendo el primer hijo con 30,6 años de media, esas trabajadoras están ya cerca de los cuarenta y cinco años, si no los han cumplido.

En base a lo expuesto, se deduce que las trabajadoras que son madres, cuando empieza a tener un mayor grado de autonomía, porque los hijos son mayores, han entrado o están a punto de entrar en esa franja de edad maldita en el mercado de trabajo español, en la que en el mejor de casos, lo tienen muy difícil ser promocionadas en sus empresas y culminar con éxito su carrera profesional, y en muchas ocasiones, más en los tiempos críticos que hemos vivido y estamos viviendo, sus empleadores han hecho un expediente de regulación de empleo y se quedan en el paro, con unas perspectivas pesimistas y quizás no se vuelvan a emplear.

De lo que se deduce que los estereotipos, en algunas ocasiones, pueden ser muy crueles para las personas, y en este país tenemos un ejemplo muy cercano, actualmente ya superado, que es el de la mujer soltera. Debido a que en la España franquista el destino de una mujer era casarse y tener hijos, la que no seguía ese camino, muchas veces por no sacrificar su libertad o compartir su vida con una persona que no le parecía adecuada, era objeto de crítica y sufría cierta presión social para que se casase. La película "Calle Mayor" dirigida por J. A. BARDEM nos muestra hasta qué punto estaban condicionadas las mujeres en esa época, incluso se les quería imponer su estado civil, y si no seguían las pautas sociales se les trataba con extrema crueldad.

Los usos sociales y laborales condicionan nuestras vidas, pero no puede ser hasta el punto de que frustren un proyecto vital o una carrera profesional. La protagonista 
de "Calle Mayor" ve limitadas todas sus salidas y al final la única opción que tiene es quedarse en ese lugar donde no tenía ningún futuro.

Respecto a lo que nos influyen el contexto en donde vivimos, según la Encuesta de Fecundidad correspondiente a 2018 y que ha publicado el INE, las dificultades para conciliar la vida familiar y laboral y los problemas económicos por los bajos salarios hacen que las españolas retrasen su maternidad una media de 5,2 años respecto a la edad en la que tenían previsto tener su primer hijo y tengan menos de los deseados ${ }^{3}$. Volvemos a lo indicado en el párrafo anterior, no nos pueden condicionar nuestra vida y limitar nuestros espacios de libertad, porque están restringiendo nuestro futuro.

Además de las circunstancias que hemos citado en los párrafos anteriores, queremos también, hacer referencia tanto a los horarios abusivos que sufren las personas trabajadoras en los centros productivos, como a los horarios irracionales de servicios médicos, colegios, etc., que hacen, por ejemplo, mucho más difícil llevar a un familiar a una consulta médica o ir a entrevistarse con el profesor o profesora de su hija e hijo.

En relación con el primer punto citado en el párrafo anterior, España está entre los países menos productivos de Europa, pese a eso también es una de las naciones del continente en que más horas se trabajan y que menos tiempo se dedica a la formación, si además hay menos flexibilidad laboral y un número muy elevado de empresas siguen teniendo la jornada partida, podemos inferir, sin lugar a dudas, de que ese contexto no facilita el equilibrio entre la vida laboral y familiar y entorpece profundamente la cualificación profesional.

Pero en los párrafos anteriores sólo hemos examinado los problemas que tienen los trabajadores por cuenta ajena, no estaríamos abordando todo el marco laboral, si no reflejáramos los problemas que tienen para conciliar las trabajadoras y los trabajadores por cuenta propia, que además han experimentado un incremento significativo en los últimos años, entre otras cuestiones, por considerarse desde la Administración que era una alternativa al desempleo. Las personas que trabajan por cuenta propia usualmente están ocupadas en su labor un número elevado de horas al día, porque además de atender los pedidos de sus clientes, tienen que llevar su administración, tratar con las entidades financieras, etc., en fin, tienen que hacer múltiples tareas para conseguir sus objetivos, sin haber un límite claro entre la vida profesional y personal, originando esta situación graves problemas de salud en las personas que desempeñan su oficio en ese colectivo.

También queremos reflejar, a los que con frecuencia no se les cita en este contexto, y seguramente tienen más dificultades que los otros dos colectivos laborales, citados en los párrafos anteriores, para poder compaginar su trabajo, con el cuidado de la familia, que son las trabajadoras y trabajadores que realizan su labor en la economía sumergida, muchas de ellas, la mayoría en el sector doméstico son mujeres, cuidando a bebes y personas mayores, para que los familiares de los mismos

3 Datos extraídos del artículo: (9 de abril de 2019). Las mujeres retrasan la maternidad más de cinco años por los bajos sueldos y la falta de conciliación. Expansión. Recuperado de http://www.expansion.com/economia/2019/04/09/5cac7146268e3ecd238b4611.html 
puedan acudir a su centro productivo. Parece olvidarse que esas mujeres también tienen hijas e hijos y parientes mayores a los que atender, por lo que necesitan tener una remuneración y unos servicios adecuados para poder desenvolverse.

De lo que se puede deducir, por lo expuesto en el desarrollo del presente artículo, que si la conciliación en la materia que estamos tratando, recae sobre todo en las trabajadoras, ellas serán las que en mayor medida sufrirán las consecuencias de esos horarios de trabajo tan inadecuados para el desarrollo de la persona, además de lo que afecte a su salud, y serán sobre las que recaigan los estereotipos tan absurdos que hemos citado en los párrafos anteriores y en definitiva las que tengan peores salarios $y$ menos retribución en sus pensiones, y también, no por citarlo en último lugar es menos importante, la frustración de no haber conseguido sus objetivos, peso a los esfuerzos tan importantes que han hecho en el periodo de formación y a las renuncias familiares para poder avanzar en su carrera profesional.

$\mathrm{Si}$, además, no hay una flexibilidad de horarios escolares, de los servicios del sistema de salud, oficinas financieras, comercio, etc. Los tramites que se tienen que realizar con las distintas administraciones que requieren la presencia física del usuario, usualmente son con cita y hora inamovible, si se modifica, la próxima cita es varios días después, y los servicios de cuidado de las personas mayores o enfermas aún distan de ser los adecuados.

De lo que es fácil concluir los horarios tan complicados que tiene la ciudadanía, tanto en el ámbito laboral como en el personal, si a esas circunstancias tan negativas le sumamos las deficiencias en los transportes colectivos, realmente tenemos que admitir que a los problemas de conciliación se une los problemas de tiempo, planteando una cuestión muy difícil de resolver.

Porque con frecuencia leemos en los periódicos o escuchamos en otros medios de comunicación los retrasos que sufren los trenes de cercanías de Madrid que, en días laborales, según el Ministerio de Fomento, utilizan más de 900.000 viajeros 4 . Igualmente, también conocemos noticias similares de otras grandes urbes de nuestro país.

Pero en municipios que tienen menos población que Madrid, como Murcia, también hay problemas en los transportes colectivos, por ejemplo, una persona para trasladarse de una pedanía de la zona sur a otra de la zona norte, que distan veinte kilómetros, tarda una hora y media, si hace dos viajes al día, ida y vuelta que es lo lógico, está tres horas en el autobús. Tres horas menos que tiene esa persona para estar con su familia, para descansar o para otras actividades. Si hiciera la jornada partida y volviera a su casa a comer, prácticamente está todo el día subida en el autobús.

Si tenemos en cuenta que, en la zona norte de la ciudad, además de varios centros comerciales y un importante número de empresas, está el campus de la Universidad pública donde están ubicadas las mayorías de las facultades y el campus de la

4 (30 de julio de 2018). Cercanías Madrid cierra el primer semestre con 130 millones de pasajeros, un 6,3\% más que el año pasado. Europa Press. Recuperado de https://www.europapress.es/madrid/noticia-cercanias-madrid-cierra-primer-semestre-130-millonespasajeros-63-mas-ano-pasado-20180730142513.html 
Universidad privada, nos podemos hacer una idea de las trabajadoras y trabajadores y estudiantes que todos los días le tienen que restar tres horas a su jornada. Igualmente, los que se desplacen de la zona norte al sur, aunque existen menos centros productivos, el sur también existe como escribió Mario Benedetti, también hay residentes de las pedanías de la costera norte que tienen su trabajo en las pedanías de la costera sur.

Hemos expuesto el caso de Murcia, por conocerlo ya que residimos en ese municipio, pero con toda seguridad, que hechos similares se darán en varias ciudades de España, porque no se está observando, en la medida que corresponde, como influyen los transportes colectivos en la conciliación de la vida laboral y familiar, además de que esas pérdidas de tiempo generan estrés, que añadido al que originan las múltiple circunstancias a las que tienen que hacer frente las personas trabajadoras diariamente, les pueden ocasionar daños en la salud de gravedad.

\section{Nuevos desafíos}

Estamos en un momento de transición hacia una nueva era económica, donde prácticas o referencias del pasado ya no son válidas y nos tendremos que adaptar continuamente a nuevas formas de proceder, porque una de las características del tiempo en que vivimos es la rápida sucesión de los acontecimientos y por tanto lo difícil que es adaptarse a los mismos.

Se desconoce cómo va a afectar al empleo, el nuevo modelo productivo que se le está denominando "economía digital", que como es lógico suscita muchas incógnitas y que pese a estar en sus inicios, ya ha tenido un fuerte impacto, entre otros, en el sector de la prensa escrita y desde hace un tiempo se están anunciando expedientes de regulación de empleo en el sector financiero, que van a afectar a un número elevado de trabajadores. A este respecto, Víctor Cruzado en el diario Expansión publica un artículo con el siguiente título «La banca hará más despidos en 2019 que en los peores años de la crisis», debido a los procesos de transformación digital5.

Lo que, si es indudable, que el mercado de trabajo que, en el caso de nuestro país, como hemos indicado anteriormente es muy difícil, se va a ver sometido a numerosos cambios, y muy probablemente, todavía incrementara más su complejidad y será más arduo acceder a empleos de calidad.

Esa tesitura, puede entorpecer los avances que se realicen en materia de igualdad entre mujeres y hombres, porque es evidente, y así consta en el importante volumen de literatura que se ha generado al respecto, que la independencia de las mujeres, al igual que la de los hombres, está estrechamente asociada a un empleo remunerado, porque esa base económica es necesaria para poder construir nuestra vida.

Además de los cambios que pueda originar, lo que también se ha denominado Tercera Revolución Industrial, estamos en una sociedad de demografía madura o envejecida, en el año 2050 los mayores de 80 años se habrán triplicado, según la socióloga María Ángeles Durán (2018), "ni el modelo actual de familia ni el actual

5 Cruzado, V. (3 de mayo de 2019). La banca hará más despidos en 2019 que en los peores años de la crisis. Expansión. Recuperado de

http://www.expansion.com/empresas/banca/2019/05/03/5ccb461c22601df23b8b460d.html 
sistema de pensiones, organización sanitaria y servicios sociales están preparados para hacer frente a este colosal desafío" (p. 104).

Igualmente, la autora citada anteriormente nos indica que:

Durante algunos años ha formado parte de un comité científico asesor en el Ministerio de Sanidad del que también formaban parte representantes de asociaciones médicas y alli se planteaba un horizonte posible de ciento veinte años de esperanza de vida a un plazo no demasiado lejano, aunque, como ella misma señala, esos horizontes de longevidad sólo son horizontes: pero no resultan descabellados y cada vez parecen más cerca. Por lo tanto, a medida que los umbrales de juventud y vejez se desplacen, la organización social basada en la edad tendrá que cambiar, asimismo, el diseño de las ciudades y el transporte tendrá que adaptarse a la creciente presencia de ciudadanos con las facultades de oír, ver y moverse ligera o profundamente disminuidas (Durán, 2016, p. 117).

Para hacer frente a ese reto, y evitar que nuestras tradicionales pirámides demográficas que se están convirtiendo en torres, no se inviertan, se están impulsando políticas para incrementar la natalidad, por lo que va a ser necesario aumentar notablemente los recursos para poder atender a esas áreas de la dependencia.

Asimismo, habrá de plantearse como se establecen las tareas de cuidado de esos dos ámbitos de la dependencia, no pueden recaer, como sigue sucediendo en la actualidad, en las mujeres, porque además de ser una tarea de envergadura y que necesitará un número de horas muy importante, lastraría los avances que tanto han costado de obtener y eliminaría todas las posibilidades de promoción profesional y realización personal de este último colectivo, repercutiendo negativamente al conjunto de la sociedad.

No se puede prorrogar la situación que tienen las mujeres en la actualidad, porque como señala Durán (2018):

Es la hija (a veces también el hijo, pero sobre todo la hija) la que cuida a su madre y a su padre en la fase de deterioro, al final de sus vidas. Es la maternidad invertida de las generaciones, siendo ahora las madres de sus madres (p. 200).

A esas madres de 35 a 54 años se les ha denominado generación "sándwich", porque son las personas que balancean las responsabilidades exigentes y delicadas de cuidar a sus hijos y a sus padres a la misma vez y según la Asociación Americana de Psicología sienten más estrés que cualquier otro grupo de mujeres ${ }^{6}$.

En base a lo expuesto en los párrafos anteriores, el equilibrio entre la vida laboral, familiar y personal va a estar muy condicionado en un futuro próximo, por los cambios que se produzcan en las relaciones laborales y por la evolución demográfica. Si en relación al ámbito laboral se desconoce en qué medida los cambios que se avecinan

\footnotetext{
6 American Psychological Association https://www.apa.org/centrodeapoyo/sandwich
} 
pueden afectar al empleo, con respecto a la comunidad, si sabemos que el número de personas mayores se incrementara notablemente y que es necesario fomentar la natalidad para garantizar la sostenibilidad del país.

Para hacer frente a ese gran problema estructural que tiene nuestro país y que se le está denominando invierno demográfico, por el aumento de la población jubilada y la caída de la natalidad, será necesario tomar medidas muy transcendentales, que con seguridad algunas serán rechazadas por la población, pero en lo que no cabe ninguna duda, es que es necesario un mercado laboral de pleno empleo y que el mismo sea de calidad, son necesarios muchos recursos y difícilmente si los niveles de desempleo son muy altos, se podrán tener los medios adecuados.

Se inicia, como hemos expuesto en los párrafos anteriores, una nueva era económica y productiva, se desconoce, como es lógico, como puede afectar al bienestar de las personas, pero lo que, si es evidente que, con los temas que nos ha dejado pendientes, lo que se ha denominado, la sociedad de la comunicación e información, no se pueden repetir las cifras de desempleo de esa época, porque además del daño que produce a las personas trabajadoras, entorpecería en gran medida los avances que necesita la ciudadanía.

\section{Reflexiones y propuestas}

El siglo XX concluyó, como es lógico, dejando pendientes muchas cuestiones que se iniciaron o se hicieron patentes en ese periodo histórico, no por pasar una hoja en un calendario se queda un problema atrás, pero al iniciar una nueva etapa se renuevan las ilusiones y se pretenden enfocar los asuntos de forma distinta para no cometer los mismos errores que en el pasado, y el inicio de un nuevo siglo, y en este caso de un nuevo milenio, nos motiva para intentar superar los temas que teníamos postergados.

Uno de los principales retos que se hizo patente en gran medida en el siglo pasado, aunque su raíz está insertada desde los orígenes de la civilización, y que aún no se ha logrado, es la igualdad real entre mujeres y hombres. Una muestra ostensible del descontento en el seno de la sociedad ante la marginación que sufren las mujeres en diversos ámbitos se hizo evidente el Día Internacional de la Mujer del año 2018 en el que miles de personas se manifestaron en 120 ciudades de España y hubo una huelga de veinticuatro horas y paros en muchas empresas, pasando a ser el ocho de marzo de ese año una fecha histórica y una referencia para lograr una sociedad igualitaria. En el año 2019 también hubo manifestaciones en muchas ciudades de nuestro país, y es de prever que esos hechos se irán sucediendo hasta que todas las personas, independientemente de su sexo, tengan la misma igualdad de oportunidades. La consecución de ese objetivo no puede seguir siendo una utopía, debe ser ya una realidad.

Y entre los obstáculos que impiden lograr el objetivo de una sociedad igualitaria, uno de los más importantes, refrendado por muchos expertos en la materia, es que la atención a la familia y el hogar no sea asumida en mayor medida por las mujeres, y a este respecto la periodista Raquel Pascual Cortés (2018) publicó un artículo con motivo del día internacional de la mujer, donde indicaba que:

La verdadera brecha de género está marcada por las necesidades de conciliación para el cuidado de hijos u otros familiares. En el citado 
artículo la autora va exponiendo las reflexiones que le han llevado a esa conclusión y entre los argumentos destaca, que de cada 13 mujeres que reducen la jornada para poder cuidar de sus hijos, solo un hombre lo hace. Esta situación de las trabajadoras, con salarios más bajos, menos horas trabajadas y cotizadas y carreras laborales intermitentes pasa factura, entre otras cuestiones, en el cobro de la pensión. De media las pensiones femeninas son un 34\% inferiores a la de los hombres.

Pero además de los datos económicos, tenemos también el factor humano, la autoestima de la persona al percibir que está contribuyendo al progreso de la sociedad y las posibilidades de ver cumplidas sus expectativas e inquietudes profesionales. Entendemos que este relato, que nos cuenta la gran escritora chilena Marcela Serrano en su obra "La Novena", una bella e inusual historia, nos puede acercar a lo que pretendemos trasladarles.

Y el primer día que sali de casa a trabajar, créeme, sentí que había amanecido varios metros más alta. Pero no se trataba de una pasión, era más bien una subida de la autoestima. Con estas palabras, intenta trasladarle Amelia a Miguel como se sentía al volver a trabajar, después de haber estado unos años sin estar empleada en un trabajo remunerado al contraer matrimonio y dedicarse a las tareas de la casa y al cuidado de los hijos.

No es extraño lo que sentía la protagonista de dicha novela, al volver a incorporarse al mercado laboral, porque el trabajo además de proporcionarnos una remuneración económica que nos debe permitir una existencia conforme a la dignidad humana, también, y no es menos importante que el aspecto financiero, puede contribuir a dar sentido a nuestras vidas y ser un pilar básico en nuestra realización como personas.

Por lo tanto, es fundamental avanzar hacia una sociedad donde todos sus integrantes tengan igualdad de oportunidades, no se puede seguir en un modelo desfasado, como indicábamos en el tercer epígrafe del presente trabajo, por lo tanto, entre otras cuestiones entendemos que sería necesario, progresar en las siguientes materias:

PRIMERA. Las tareas y las responsabilidades familiares se deben de repartir de forma equilibrada entre todos los miembros de la familia, para que no sean asumidas, como ocurre en la mayor parte de los casos, por las mujeres. Debe haber una corresponsabilidad en las labores que origina el hogar y en las decisiones que hay que tomar al respecto para lograr la igualdad entre mujeres y hombres.

SEGUNDA. Las Administraciones Públicas tienen que implicarse totalmente en la conciliación de la vida laboral y familiar, no es concebible, que el Estado español en el año 2015 solo haya financiado el $6 \%$ de los servicios de cuidado que reciben los menores de tres años, cuando países como Suecia y Dinamarca financian el $100 \%$ de las plazas de guardería, según el estudio "Focus on Spanish Society" de la Fundación de Cajas de Ahorros (Funcas) publicado en el año 20187. Probablemente, por esa

Datos extraídos del artículo: Bolaños, A. (7 de marzo de 2018). España se sitúa a la cola de la Unión Europea en oferta de guarderías públicas. El País. Recuperado de 
atención tan especial a las familias, los países nórdicos, aunque no son todo lo idílicos que creíamos, "tienen una tasa de fecundidad de 1,9 hijos por mujer, las parejas tienen los hijos a edades más jóvenes, con lo que la brecha generacional a la hora de educarlos es menor, que siempre beneficia a ambos" (Das Gupta et al., 2014, p. 111).

Además, como hemos expuesto en el desarrollo del trabajo, entendemos que, para avanzar en conciliación, la tendencia debe ser que las tareas de cuidado en una parte importante se externalicen y que en su mayor parte las asuma el estado, porque es quien dispone de mayores recursos financieros y puede garantizar mejor dicha labor.

TERCERA. Este punto se encuentra estrechamente relacionado con el anterior. Para externalizar las tareas de cuidado, que no pueden asumir las familias, es imprescindible que las personas que realizan ese trabajo tengan el reconocimiento que merece esta difícil labor, tanto económico como laboral, con la formación adecuada y las remuneraciones y las condiciones de trabajo adecuadas. Son las personas que se ocupan de lo que más nos importa, por lo tanto, nos debe interesar mucho el grado de satisfacción en su trabajo. Además, se crea un nicho de trabajo donde se van a emplear un número significativo de trabajadores, porque como hemos expuesto en el presente artículo, se va a incrementar notablemente el número de personas dependientes $y$, en consecuencia, la contribución fiscal del nuevo sector de empleo, aumentaran las cotizaciones a la seguridad social y la recaudación de impuestos.

Un mercado de trabajo con empleos de calidad genera más empleos de calidad y mayor bienestar a la población, un mercado laboral precario crea más precariedad y hace más complicada la vida de la ciudanía. Esa ecuación es fácil de exponer, pero lo que resulta muy difícil es llegar a ella, parece una utopía cuando es una realidad demostrada ya en otros países.

CUARTA. El punto cuarto está estrechamente enlazado con el último párrafo del anterior. Porque como hemos expuesto, la precariedad laboral, limita todas las posibilidades de las personas, tanto en el ámbito público como en el privado. En relación con la materia que estamos tratando, unos salarios tan bajos no permiten ni reducir la jornada, ni externalizar tareas de cuidado y por lo tanto afectan negativamente a la calidad de vida de las trabajadoras y de los trabajadores y de sus familias. Asimismo, estas personas tienen escasa capacidad para financiar el cuidado remunerado y en consecuencia cuando pueden externalizar alguna labor de cuidado, con frecuencia no pueden tratar a los trabajadores que van a realizar ese cometido con una remuneración adecuada y la protección social correspondiente, ampliando de esta forma el circulo de la precarización, como indicábamos en la cuestión anterior.

QUINTA. Si de lo expuesto en el desarrollo de artículo, se deduce con mucha claridad, las dificultades tan patentes que existen para lograr el equilibrio entre la vida laboral, familiar y personal y las barreras tan elevadas que hay para poder conseguir ese objetivo, se puede inducir sin ninguna cortapisa en qué situación están al respecto las responsables y los responsables de las familia monomarentales y monoparentales, indicamos en primer lugar monomarentales porque un noventa por ciento de las mismas están formadas por madres solteras. Esas personas además de tener más

https://elpais.com/economia/2018/03/06/actualidad/1520354611_098547.html 
dificultades para encontrar un empleo, porque las discriminan por tener cargar familiares, tienen que hacer frente solas a las tareas que requiere el hogar familiar y disponen de menos recursos económicos para externalizar la labor de cuidado, por lo que ese colectivo debía recibir más apoyo de la Administración.

SEXTA. Reflejábamos en el tercer epígrafe, que un 73\% de empleados en España, manifiesta que su entorno laboral en lugar de facilitarles la conciliación entre la vida laboral y familiar, la dificulta ocasional o sistemáticamente. Si además tenemos en cuenta, que el tejido productivo de nuestro país está formado mayoritariamente por pequeñas y medianas empresas, en la que muchos empresarios no están sensibilizados con la materia objeto de estudio, podemos entender que ese índice es más elevado y es necesario que las políticas de igualdad tengan presencia en ese sector mercantil. En este sentido, es muy significativa las medidas que aplican las grandes empresas para favorecer la conciliación de sus empleados, porque pueden ser una referencia de buenas prácticas, por la gran difusión que tienen.

Finalizamos, indicando que las medidas descritas, son sólo algunas de las muchas que se tendrían que analizar y poner en práctica para avanzar en esta materia tan fundamental en el bienestar de la ciudadanía. Iniciamos un nuevo siglo, un nuevo milenio, una nueva era productiva, son muchos desafíos, y no podemos repetir los mismos errores que en el pasado reciente, cometeremos otros, sin ninguna duda, pero las consecuencias de no haber tenido las políticas y las medidas adecuadas, en estas últimas cinco décadas, ya las conocemos y debemos superarlas.

Queremos concluir, que es difícil acercarse al tema de la conciliación, y plantear puntos sobre lo que no hayan escrito otros autores, ya que es una materia sobre la que se ha generado mucha literatura, pero sobre las cuestiones analizadas con más frecuencia por otros investigadores, hemos intentado tratarlas desde otra perspectiva, con el ánimo de poder realizar una aportación de utilidad y asimismo, entendemos que es importante como puede afectar el empleo del futuro al equilibrio entre la vida laboral, personal y familiar, ya que estamos ante un tema sociolaboral muy complejo, donde la relación trabajo y sociedad es mucho más intensa que en otros aspectos sociales y laborales.

\section{Bibliografía}

Álvarez, R. J. (7 de marzo de 2018). El 36\% de los hombres deja las tareas del hogar al vivir en pareja. El Mundo. Recuperado de https://www.elmundo.es/espana/2018/03/07/5agfo34d22601dco3e8b4592.htm

Bolaños, A. (7 de marzo de 2018). España se sitúa a la cola de la Unión Europea en oferta de guarderías públicas. El País. Recuperado de https://elpais.com/economia/2018/03/06/actualidad/1520354611_098547.html

Cruzado, V. (3 de mayo de 2019). La banca hará más despidos en 2019 que en los peores años de la crisis. Expansión. Recuperado de http://www.expansion.com/empresas/banca/2019/05/03/5ccb461c22601df23b 8b46od.html

Das Gupta, M., Engelman, R., Levy, J., Luchsinger, G., Merrik, T. y Rosen, J. E. (2014). El Estado de la Población Mundial 2014. El poder de 1.800 millones. Los adolescentes, los jóvenes y la transformación del futuro. UNFPA. Recuperado de 
http://estaticos.elmundo.es/documentos/2014/11/18/onu_estado_de_la_pobla cion.pdf.

Durán, M. A. (2015). El nuevo rostro de las familias españolas. Dendra Médica. Revista de Humanidades, 14(2), 39-54. Recuperado de http://www.dendramedica.es/revista/v14n2/11_El_nuevo_rostro_de_las_famili as_espanolas.pdf

Durán, M. A. (2016). El futuro del cuidado. El envejecimiento de la población y sus consecuencias. Pasajes. Revista de Pensamiento Contemporáneo. (Ejemplar dedicado al inquietante Siglo XXI). (50), 114-127. Recuperado de http://digital.csic.es/bitstream/10261/147623/1/El\%2ofuturo\%2odel\%2ocuidad o_Angeles\%2oDuran.pdf

Durán, M. A. (2018). La riqueza invisible del cuidado. Universidad de Valencia.

Freire, E. (2008). Mucha demagogia. Psychologies, N. ${ }^{\circ} 40$.

Las Heras, M. (2018). Estudio Responsabilidad Familiar Corporativa (IFREI) España 2017: Consecuencias en las empresas, los empleados, y la sociedad, IESE, ST-485, $10 / 2018$

Pascual Cortés, R. (8 de marzo de 2018). ¡Es la conciliación, estúpidos!». Cinco Días. Recuperado de https://cincodias.elpais.com/cincodias/2018/03/07/midinero/1520446972_3219 51.html

Valls-Llobet, C. (2010). Mujeres, salud y poder. Ediciones Cátedra. Universitat de Valencia. Instituto de la Mujer, $2^{\text {a }}$ edición.

\section{Cómo referenciar este artículo/How to reference this article:}

Uris Lloret, J. M. (2020). Vigésimo aniversario de la Ley de conciliación de la vida laboral y familiar. iQUAL. Revista de Género e Igualdad, 3, 46-61, doi: 10.6018/iqual.382821

Uris Lloret, J. M. (2020). Vigésimo aniversario de la Ley de conciliación de la vida laboral y familiar. [Twentieth anniversary of the law of conciliation of labor and family life]. iQUAL. Revista de Género e lgualdad, 3, 46-61, doi: 10.6018/iqual.382821 